\title{
Fluctuation spectrum of membranes with anchored linear and star polymers
}

\author{
Thorsten Auth* and Gerhard Gompper \\ Institut für Festkörperforschung, Forschungszentrum Jülich, D-52425 Jülich, Germany \\ (Received 22 February 2005; revised manuscript received 1 July 2005; published 13 September 2005)
}

\begin{abstract}
The effect of linear homopolymers, diblock copolymers, and star polymers anchored to a membrane on the membrane's fluctuation spectrum is investigated for low grafting densities. Due to the nonlocality of the polymer-membrane interaction, the effective bending rigidity $\kappa_{\text {eff }}(\mathbf{q})$ of the composite membrane is found to depend strongly on the wave vector $\mathbf{q}$ of the membrane undulations. Analytical calculations for ideal linear chains and simulations for ideal and self-avoiding linear chains as well as for star polymers are presented. The analytical calculations are based on the Green's function approach of Bickel and Marques [Eur. Phys. J. E 9, 349 (2002)]; for the simulations the Monte Carlo method is used. The functional form of $\kappa_{\text {eff }}(\mathbf{q})$ differs for end-grafted chains and diblock copolymers. In general, the polymer effect is most pronounced for undulations on length scales larger than or comparable to the polymer size, and decreases rapidly for smaller undulation wavelengths. Anchored linear chains always increase $\kappa$; anchored star polymers may increase as well as decrease $\kappa$, depending on whether they are anchored symmetrically or asymmetrically to the membrane.
\end{abstract}

DOI: 10.1103/PhysRevE.72.031904

PACS number(s): 87.16.Dg, 36.20.-r, 82.70.-y

\section{INTRODUCTION}

The polymer-membrane interaction is of interest in many soft matter, biomimetic, and biological systems like microemulsions [1], lamellar membrane stacks [2], stealth liposomes [3], and red blood cells [4-6]. In these systems, polymers can be attached to membranes in various ways. For example hydrophilic chains can be anchored at one end by a hydrophobic anchor in a lipid membrane [3,7] and amphiphilic diblock copolymers will assemble at a surfactant monolayer of an oil-water mixture $[1,8,9]$. Triblock copolymers can bind with one block in a surfactant bilayer [10] and polymers can adsorb at a membrane [11]. Depending on the number of polymers per membrane area, a high- and a lowdensity regime with fundamentally different physical behavior can be distinguished. For high grafting densities above the overlap concentration the chains have to stretch away from the membrane and form polymer brushes $[12,13]$. We will focus on the low-density regime, in which the polymer grafting density is smaller than the overlap density and the average distance between the polymers is larger than their average size. In this case, the monomer density of a linear end-grafted chain has a mushroomlike shape and each polymer exerts a pressure on the membrane, which decreases with increasing distance from the anchor point [14-18]. This implies a deformation of the membrane into a locally conelike "pinch" shape [14-18].

If the polymer size is much smaller than the mean structural size it is often convenient to model compound membrane-polymer systems by homogeneous elastic surfaces and to take into account the polymer effect by effective curvature elastic constants. Thus the Hamiltonian of the compound system has the same functional form as the Hamiltonian of a pure membrane system [19-21],

*Present address: Weizmann Institute of Science, Department of Materials and Interfaces, Rehovot 76100, Israel.

$$
\mathcal{H}=\int d S\left(\frac{\kappa}{2}\left(c_{1}+c_{2}-2 c_{0}\right)^{2}+\bar{\kappa} c_{1} c_{2}\right)
$$

where $c_{1}$ and $c_{2}$ are the principal curvatures at each point of the membrane. Here, $\kappa$ is the bending rigidity, $\bar{\kappa}$ the saddlesplay modulus, and $c_{0}$ the spontaneous curvature. The integral is performed over the whole membrane area. This strategy allows us to use the well-developed knowledge on the physical behavior of pure membranes to investigate new effects which occur in compound systems. It has been applied successfully to understand the "efficiency-boosting" effect of amphiphilic block copolymers in ternary microemulsions $[8,9]$.

In the limit of large fluctuation wavelengths, the polymer effect can be calculated by using expansions of the free energy in powers of the curvature. The expansion coefficients for polymers anchored to spherically and cylindrically deformed walls can be related to the elastic constants. With this method, the effects of polymer self-avoidance and of the star polymer architecture have recently been studied by computer simulations [22]. For ideal linear chains anchored to a membrane, analytical calculations are also available $[23,24]$.

A polymer affects the properties of the membrane not only at the location of its anchor, but on an area proportional to the square of its average radius. This implies that there must be in general a nonlocal interaction between different membrane points. Under the assumption of small fluctuations, the Monge representation for the membrane conformation can be used, where the membrane deformation is described by a height field $h(\boldsymbol{\rho})$, with $\boldsymbol{\rho}=(x, y)$, from a planar reference state. Thus there is a nonlocal interaction $w(\boldsymbol{\rho}$ $-\boldsymbol{\rho}^{\prime}$ ) between the height variables. The deformation energy

$$
E_{\text {int }}=\frac{1}{2} \int d \boldsymbol{\rho} \int d \boldsymbol{\rho}^{\prime} w\left(\boldsymbol{\rho}-\boldsymbol{\rho}^{\prime}\right)\left[h(\boldsymbol{\rho})-h\left(\boldsymbol{\rho}^{\prime}\right)\right]^{2}
$$

can be expressed in terms of the undulation modes 


$$
h(\mathbf{q})=\int_{-\infty}^{\infty} d \boldsymbol{\rho} h(\boldsymbol{\rho}) e^{-i \mathbf{q} \cdot \boldsymbol{\rho}}
$$

with wave vector $\mathbf{q}$ as

$$
E_{\text {int }}=\int \frac{d \mathbf{q}}{(2 \pi)^{2}} h(\mathbf{q}) h(-\mathbf{q})[w(\mathbf{0})-w(\mathbf{q})] .
$$

Since $w(\mathbf{0})-w(\mathbf{q}) \sim q^{4}$ is found for $|\mathbf{q}| \rightarrow 0$, a length-scaledependent effective bending rigidity $\kappa_{\text {eff }}(\mathbf{q})$ can be defined.

The fluctuation amplitude of the bare membrane decreases with the fourth power of the wave vector of the undulation modes,

$$
\left\langle|h(\mathbf{q})|^{2}\right\rangle \propto \frac{1}{\kappa q^{4}} .
$$

Therefore, it is intuitively clear that the effect of membrane undulations on the polymer conformations becomes less important with increasing wave vector; the polymer conformations are little affected by small ripples on the membrane. This behavior has recently been obtained quantitatively in Ref. [25] from Green's function calculations for a membrane decorated with ideal end-grafted linear chains. We apply the same analytical method here to ideal diblock copolymers. The analytical results are compared with results of Monte Carlo simulations, which we have performed for both cases. Monte Carlo simulations are also used to study the effects of self-avoiding polymer chains on the curvature elasticity. In particular, we investigate linear end-grafted chains, diblock copolymers, and star polymers with excluded-volume interactions. The case of self-avoiding diblocks corresponds to the experimental system of amphiphilic block copolymers in microemulsions (see Refs. [8,9,26]).

\section{MODEL FOR FLEXIBLE POLYMERS AT MEMBRANES}

A system with an almost planar membrane and one attached polymer is considered. The simulation snapshot of a symmetric diblock copolymer in Fig. 1 illustrates the model. The spontaneous curvature $c_{0}$ is chosen to be zero. Due to the Gauss-Bonnet theorem, the term with the saddle-splay modulus is a topological invariant and can therefore be omitted [27]. For small deviations $h(\boldsymbol{\rho})$ from a planar reference state, the membrane Hamiltonian in Eq. (1) simplifies to

$$
\mathcal{H}=\frac{\kappa}{2} \int d \boldsymbol{\rho}\left[\nabla^{2} h(\boldsymbol{\rho})\right]^{2} .
$$

Thus, we calculate here the polymer effect on the bending rigidity $\kappa$; its influence on the other elastic constants will not be discussed. Note that our simulation results for $\kappa_{\text {eff }}(\mathbf{q})$ are based on wave vectors $\mathbf{q}$ that all point in the same direction. This approach is justified as long as undulation modes with different wave vectors $\mathbf{q}$ do not couple. ${ }^{1}$.

\footnotetext{
${ }^{\mathrm{I}}$ The calculation of the effective bending rigidities with the Green's function method in Sec. III confirms this assumption. Also, a few control simulations with a two-dimensional set of wave vectors show no significant deviations.
}

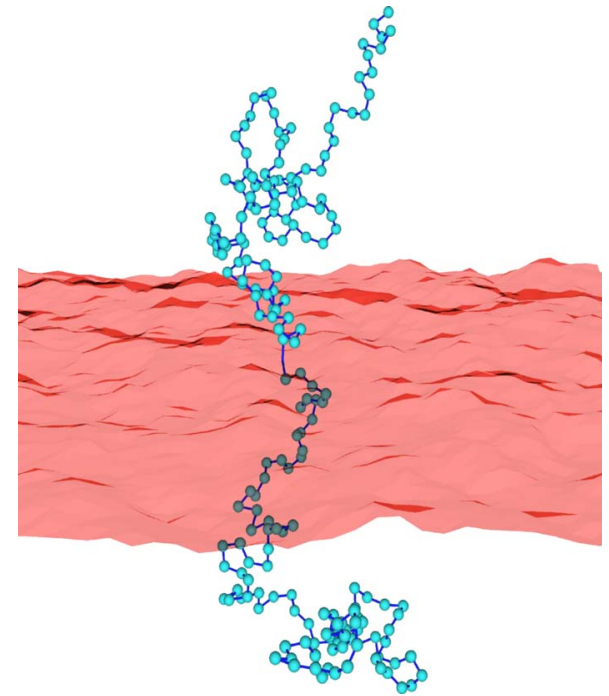

FIG. 1. (Color online) Simulation snapshot of a symmetric selfavoiding diblock copolymer anchored to a fluctuating membrane. The polymer's vertices are not allowed to cross the membrane. Beads with a mutual excluded-volume interaction mimic self-avoidance.

In the mushroom regime, the total polymer effect is linearly proportional to the grafting density $\sigma$ and the meansquared end-to-end radius $R_{e}^{2} \propto N^{2 v}$ or the squared radius of gyration $R_{g}^{2}[23,24]$. For ideal chains, $\nu=1 / 2$ and $R_{g}^{2}=R_{e}^{2} / 6$, while for self-avoiding chains, $\nu=0.588$ and $R_{g}^{2}=R_{e}^{2} / 6.25$ [28].

In the Monte Carlo simulations, the membrane is discretized on a square grid with lattice constant $a$; periodic boundary conditions are applied. It is important, however, to use continuous height variables $h_{i}$ [29]. The polymer is modeled by a freely jointed chain with bonds of length $r_{\text {bond }}=a$ $=1$. To take self-avoidance into account, beads are attached to the vertices which mutually exhibit excluded-volume interactions. The interaction between polymer and membrane is such that the polymer's vertices are not allowed to cross the membrane.

\section{THEORY FOR IDEAL, LINEAR POLYMERS}

\section{A. Clapper model}

A simple model for a short rod like polymer can easily be evaluated analytically and is useful to demonstrate the idea of the effective bending rigidity, as well as to test the code used for the simulations. The polymer is modeled by two clappers attached to the same point in the center of a membrane stripe, one clapper on each side of the membrane. In Fig. 2, the system's architecture is sketched for the case of three stripes. The Hamiltonian of a bare membrane of size $L \times L$ with periodic boundary conditions and fluctuating only in one direction reads

$$
\mathcal{H}_{\text {mem }, L}=\frac{\kappa}{2} L \sum_{i=1}^{L}\left(\frac{h_{i-1}+h_{i+1}-2 h_{i}}{a^{2}}\right)^{2}
$$

where $h_{i}$ are the height variables and $a$ is the stripe width (compare Fig. 3). If the clappers are located at position $i$, the 


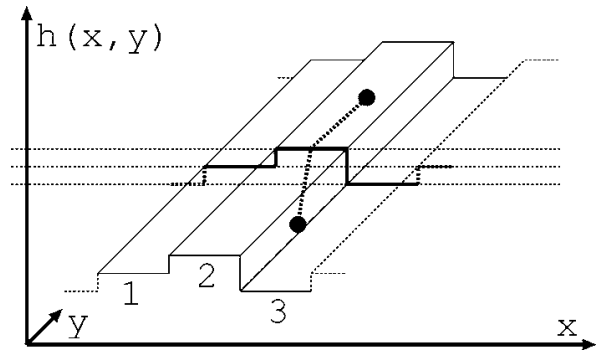

FIG. 2. Illustration of the clapper model. The membrane deformation varies only in the $x$ direction. In a solid-on-solid model, three height variables $h_{i}$ are shown. Periodic boundary conditions are employed. The membrane is impenetrable to the end points of the clapper pair, which is anchored to the center of the middle stripe.

steradian accessible to each clapper can be expressed in terms of the height differences between neighboring stripes. We use spherical coordinates with the polar axis in the $z$ direction and angles $\phi$ (parallel to the stripe the clapper is anchored to) and $\theta$ to integrate over all possible clapper orientations. With the clapper length chosen to equal the stripe width, the steradians $\Omega_{+(-)}$accessible to the clapper "above" ("below") the membrane read

$$
\Omega_{i, \pm}=2 \pi \mp \sum_{j=-1,1} \int_{0}^{\theta_{i, j}} d \theta \cos (\theta) \phi(\theta)
$$

where $\phi(\theta)=2 \arccos [1 /(2 \cos \theta)]$ and $\theta_{i, j}=\arcsin \left[\left(h_{i+j}\right.\right.$ $\left.\left.-h_{i}\right) / a\right]$. Here, $\theta_{i, j}$ are the maximum (minimum) angles for which the $\phi$ integration is partially blocked or additionally accessible due to the neighboring stripes. Note that the clappers only interact with the membrane at their end points. After expansion of the integrand for small $\theta$ and thus for small height differences between neighboring stripes,

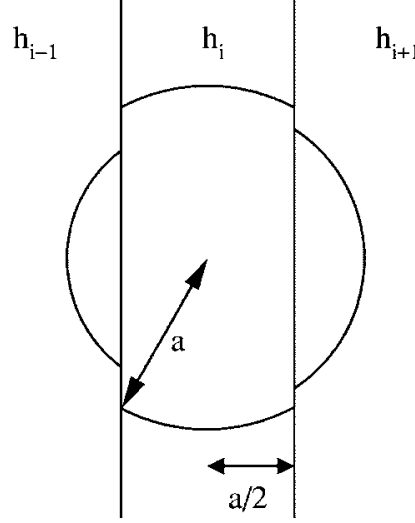

(a)
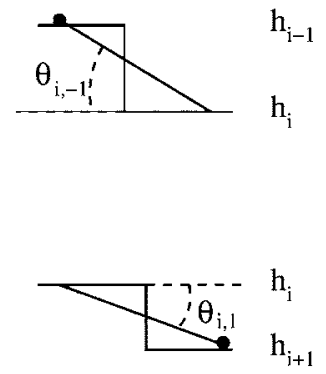

(b)
FIG. 3. Illustration of the steradian accessible to the clapper. (a) The clapper length is chosen to equal the stripe width. The circles symbolize the points where the clapper touches the stripes. (b) Due to the height differences, the circles on the neighboring stripes have the radii $a \cos \left(\theta_{i,-1}\right)$ and $a \cos \left(\theta_{i, 1}\right)$. The minimum and maximum angles for which the fluctuations affect the clappers are given by $\theta_{i,-1}$ and $\theta_{i, 1}$.
TABLE I. Analytical (second column) and simulation results (third to fifth column) for the clapper model. The error is of the order of magnitude of the last digit. Simulations have been performed for membranes with different bare $\kappa$ values.

\begin{tabular}{ccccc}
\hline \hline$L$ & $2 \sigma / 9$ & $\kappa=1$ & $\kappa=10$ & $\kappa=20$ \\
\hline 2 & 0.055 & 0.054 & 0.055 & 0.048 \\
3 & 0.025 & 0.023 & 0.026 & 0.023 \\
4 & 0.013 & 0.013 & 0.013 & 0.012 \\
\hline \hline
\end{tabular}

$$
\cos (\theta) \phi(\theta)=\frac{\pi}{3}-\frac{\pi+\sqrt{3}}{6} \theta^{2}+O\left(\theta^{4}\right),
$$

the steradians can easily be evaluated to be

$$
\Omega_{i, \pm}=2 \pi \mp \frac{2 \pi}{3} \sum_{j=-1,1}\left(\frac{h_{i+j}-h_{i}}{a}\right)+O\left(\left(\frac{h_{i+j}-h_{i}}{a}\right)^{3}\right) .
$$

Thus the ratio of the partition functions $\mathcal{Z}_{f}$ and $\mathcal{Z}_{p}$ of the clapper pair at a single site $i$ attached to a fluctuating and planar membrane, respectively, is given by

$$
\frac{\mathcal{Z}_{f}}{\mathcal{Z}_{p}}=\frac{\Omega_{i,+} \Omega_{i,-}}{4 \pi^{2}}=1-\frac{1}{9 a^{2}}\left(h_{i-1}+h_{i+1}-2 h_{i}\right)^{2}
$$

up to second order in the height field. This ratio of partition functions is averaged over the possible anchor points on all stripes. The corresponding free-energy difference of clappers anchored to fluctuating and planar membranes is then obtained as

$$
\mathcal{F}_{f}-\mathcal{F}_{p}=k_{B} T \frac{1}{L^{2}} \frac{L}{9} \sum_{i=1}^{L}\left(\frac{h_{i+1}+h_{i-1}-2 h_{i}}{a^{2}}\right)^{2} .
$$

The total free energy of the membrane-clapper system is the sum of Eqs. (7) and (12), where the excess free energy of the clappers can be interpreted as an extra contribution to the membrane bending rigidity. Thus the whole system can be characterized by an effective bending rigidity

$$
\kappa_{\mathrm{eff}}=\kappa+\frac{2 k_{B} T}{9} \sigma a^{2}
$$

where $\sigma=1 /(L a)^{2}$ is the clapper-pair number density. The analytical result for the extra contribution $\Delta \kappa / k_{B} T$ $=(2 / 9) \sigma a^{2}$ is confirmed by simulations (compare Table I). The simulation code for the clapper model forms the basis for a more general code, which is employed in Sec. IV for polymer chains with many bonds.

\section{B. End-grafted chains}

The calculation of $\kappa_{\text {eff }}(\mathbf{q})$ for flexible, end-grafted polymer chains is based on the diffusion equation for the partition function of the polymer, with the constraint that its solution vanishes at the membrane. We follow here the method sketched in Ref. [25] and presented in more detail in Ref. [30]. The only difference compared to Ref. [30] is that we 
choose the origin of the coordinate system such that the anchor point lies always in the reference plane. The advantage of this choice is that the perturbation expansion introduced below simplifies, since the orders of the perturbation correspond to the powers in $h$.

For a linear polymer anchored with one end next to a planar hard wall, the propagator reads [31]

$$
\begin{aligned}
G_{N}^{(0)}\left(\mathbf{r}, \mathbf{r}^{\prime}\right)= & \frac{3}{2 \pi N a^{2}} e^{-3\left[\left(x-x^{\prime}\right)^{2}+\left(y-y^{\prime}\right)^{2}\right] /\left(2 N a^{2}\right)}\left(e^{-3\left(z-z^{\prime}\right)^{2} /\left(2 N a^{2}\right)}\right. \\
& \left.-e^{-3\left(z+z^{\prime}\right)^{2} /\left(2 N a^{2}\right)}\right)
\end{aligned}
$$

where $\mathbf{r}$ are the coordinates of the anchor point and $\mathbf{r}^{\prime}$ the coordinates of the free end of the polymer. Integration over all possible positions of the free end leads to the partition function

$$
Z_{N}^{(0)}(\mathbf{r})=\operatorname{erf}\left(\frac{3^{1 / 2} z}{2^{1 / 2} R_{e}}\right)
$$

To prevent the partition function from vanishing as it would do in the limit $z \rightarrow 0$, a finite anchor length $z=d$ is introduced. The effect of the fluctuations can then be handled with a perturbation expansion $[25,32]$,

$$
Z_{N}=Z_{N}^{(0)}+Z_{N}^{(1)}+Z_{N}^{(2)}+\cdots
$$

where the order of the perturbation is given by the number of (explicit) interaction points with the membrane. Different orders are connected via the boundary condition $Z(\boldsymbol{\rho}, h(\boldsymbol{\rho}))$ $=0$, where

$$
\begin{aligned}
Z_{N}(\boldsymbol{\rho}, h(\boldsymbol{\rho}))= & Z_{N}^{(0)}(\boldsymbol{\rho}, 0)+Z_{N}^{(1)}(\boldsymbol{\rho}, 0)+h \frac{\partial Z_{N}^{(0)}}{\partial z}(\boldsymbol{\rho}, 0)+Z_{N}^{(2)}(\boldsymbol{\rho}, 0) \\
& +h \frac{\partial Z_{N}^{(1)}}{\partial z}(\boldsymbol{\rho}, 0)+\frac{h^{2}}{2} \frac{\partial^{2} Z_{N}^{(0)}}{\partial z^{2}}(\boldsymbol{\rho}, 0)+\cdots
\end{aligned}
$$

As mentioned above, if the membrane height at the interaction point is measured relative to the height of the anchor point, the power in $h$ corresponds to the number of interaction points. The first-order term vanishes after an average of the anchor position over the whole membrane. For the averaged partition function of an end-grafted chain we find

$$
\begin{aligned}
\left\langle Z_{N}^{(0)}+Z_{N}^{(1)}+Z_{N}^{(2)}\right\rangle= & \frac{6^{1 / 2} d}{\pi^{1 / 2} R_{e}}+\frac{6^{1 / 2}}{\mathcal{S}} \int \frac{d \mathbf{q}}{(2 \pi)^{2}} h(\mathbf{q}) h(-\mathbf{q}) \\
& \times\left(\frac{d}{2 \pi^{1 / 2} R_{e}^{3}}\left(1-e^{-q^{2} R_{e}^{2} / 6}\right)-\frac{q^{2} d}{\pi^{1 / 2} R_{e}}\right)
\end{aligned}
$$

where $\mathcal{S}$ is the membrane area, in agreement with the results of Ref. [25]. Note that the average of $Z_{N}^{(0)}$ also gives a term proportional to $h(\mathbf{q}) h(-\mathbf{q})$, since the averaging integral is performed over the real membrane surface and not over the $x-y$ plane. Details of the calculation can be found in Appendix A. The effective bending rigidity can then be extracted from the free energy of membrane undulations,
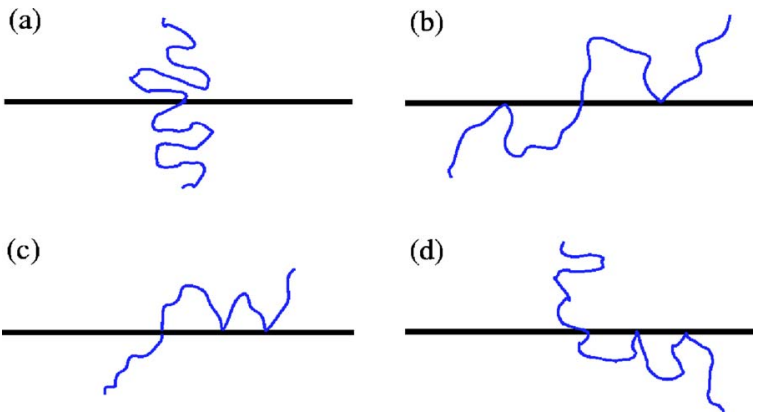

FIG. 4. (Color online) Illustrations of the terms contributing to the analytical calculations for diblock copolymers.

$$
\mathcal{F}_{f}-\mathcal{F}_{p}=-k_{B} T \ln \frac{Z_{f}}{Z_{p}}=-k_{B} T \frac{\left\langle Z_{0}+Z_{1}+Z_{2}\right\rangle-Z_{p}}{Z_{p}}+O\left(d^{2}\right)
$$

where $\mathcal{F}_{f}$ and $\mathcal{F}_{p}$ are the free energies of fluctuating and planar membranes, respectively, and $Z_{f}$ and $Z_{p} \equiv Z_{N}^{(0)}$ the corresponding partition functions. The excess free energy (6) is compared with the Fourier-transformed membrane Hamiltonian of Eq. (6),

$$
\mathcal{H}=\frac{1}{2} \int \frac{d \mathbf{q}}{(2 \pi)^{2}} \kappa_{\text {eff }}(\mathbf{q}) q^{4}|h(\mathbf{q})|^{2}
$$

with an effective, wave-vector-dependent bending rigidity $\kappa_{\mathrm{eff}}(\mathbf{q})=\kappa+\Delta \kappa(\mathbf{q})$. For $N_{p}$ noninteracting polymer chains with grafting density $\sigma=N_{p} / \mathcal{S}$, the resulting change in the bending rigidity is found to be $\Delta \kappa=a_{\kappa} k_{B} T \sigma R_{e}^{2}$ with the dimensionless amplitude

$$
a_{\kappa}=\frac{36}{q^{4} R_{e}^{4}}\left(e^{-q^{2} R_{e}^{2} / 6}-1\right)+\frac{6}{q^{2} R_{e}^{2}} .
$$

\section{Symmetric diblock copolymers}

For symmetric diblock copolymers, partition function expansions for the chain above the membrane, $Z_{N,+}$, as well as for the chain below the membrane, $Z_{N,-}$, are needed. The total partition function is the product of both expansions, corresponding to two end-grafted polymers anchored to the same point on either side of the membrane. Again the terms linear in $h$ vanish if the position of the anchor point is averaged over the membrane area; the remaining terms up to order $h^{2}$ are sketched in Fig. 4. The total averaged partition function is obtained to be

$$
\begin{aligned}
\left\langle Z_{N,+} Z_{N,-}\right\rangle= & \frac{6 d^{2}}{\pi R_{e}^{2}}+\frac{1}{\mathcal{S}} \int \frac{d \mathbf{q}}{(2 \pi)^{2}} h(\mathbf{q}) h(-\mathbf{q}) \\
& \times\left(\frac{36 d^{2}}{\pi R_{e}^{4}}\left(1-e^{-q^{2} R_{e}^{2} / 6}\right)-\frac{6 q^{2} d^{2}}{\pi R_{e}^{2}}-\frac{q^{4} d^{2}}{4}\right. \\
& \left.\times e^{-q^{2} R_{e}^{2} / 6}\left[I_{1}\left(q^{2} R_{e}^{2} / 12\right)+I_{0}\left(q^{2} R_{e}^{2} / 12\right)\right]^{2}\right),
\end{aligned}
$$

where $I_{0}$ and $I_{1}$ are Bessel functions of the first kind. Note 
that compared to the result (21) for end-grafted chains, there is an additional contribution corresponding to Fig. 4(b). Details of the calculation can be found in Appendix B. The free-energy difference

$$
\mathcal{F}_{f}-\mathcal{F}_{p}=-k_{B} T \frac{\left\langle Z_{N,+} Z_{N,-}\right\rangle-Z_{p}^{2}}{Z_{p}^{2}}+O\left(d^{4}\right)
$$

leads to the wavelength-dependent bending rigidity $\Delta \kappa$ $=a_{\kappa} k_{B} T \sigma R_{e}^{2}$ with the dimensionless amplitude

$$
\begin{aligned}
a_{\kappa}= & \frac{72}{q^{4} R_{e}^{4}}\left(e^{-q^{2} R_{e}^{2} / 6}-1\right)+\frac{12}{q^{2} R_{e}^{2}}+\frac{\pi}{2} e^{-q^{2} R_{e}^{2} / 6}\left[I_{1}\left(\frac{q^{2} R_{e}^{2}}{12}\right)\right. \\
& \left.+I_{0}\left(\frac{q^{2} R_{e}^{2}}{12}\right)\right]^{2}
\end{aligned}
$$

for chains with a bulk radius of gyration $R_{e}$ on both sides of the membrane. Thus not only is the maximum amplitude of the polymer effect different from the case of end-grafted chains, but so also is its functional dependence on the wave vector $\mathbf{q}$.

\section{Asymmetric diblock copolymers}

The calculation described above can easily be extended to diblock copolymers with two different polymerization indices $N$ and $M$ of the two blocks. The limiting cases of endgrafted and symmetric diblock chains are recovered for $M$ $=0$ and $N$, respectively. In the general case, the amplitude $a_{\kappa}$ not only depends on the sum of the radii of gyration of both chains, but displays a more complicated dependence on the chain lengths,

$$
\begin{aligned}
\frac{\Delta \kappa}{k_{B} T}= & \sigma\left(R_{e, M}^{2}+R_{e, N}^{2}\right)\left\{\frac { 6 } { q ^ { 4 } ( R _ { e , M } ^ { 2 } + R _ { e , N } ^ { 2 } ) } \left(\frac{1}{R_{e, M}^{2}}\left(e^{-q^{2} R_{e, M}^{2} / 6}-1\right)\right.\right. \\
& \left.+\frac{1}{R_{e, N}^{2}}\left(e^{-q^{2} R_{g, N}^{2} / 6}-1\right)\right)+\frac{2}{q^{2}\left(R_{e, M}^{2}+R_{e, N}^{2}\right)} \\
& +\frac{\pi}{12} \frac{R_{e, N} R_{e, M}}{\left(R_{e, N}^{2}+R_{e, M}^{2}\right)} e^{-\left(q^{2} / 2\right)\left(R_{e, N}^{2}+R_{e, M}^{2}\right) / 6}\left[I_{1}\left(\frac{q^{2}}{12} R_{e, N}^{2}\right)\right. \\
& \left.\left.+I_{0}\left(\frac{q^{2}}{12} R_{e, N}^{2}\right)\right]\left[I_{1}\left(\frac{q^{2}}{12} R_{e, M}^{2}\right)+I_{0}\left(\frac{q^{2}}{12} R_{e, M}^{2}\right)\right]\right\} .
\end{aligned}
$$

In the low- $q$ limit, the ratio between the symmetric diblock copolymer with $N$ bonds on each side and an end-grafted chain with $N$ bonds is found to be

$$
\frac{\Delta \kappa_{\mathrm{sdb}}}{\Delta \kappa_{\mathrm{eg}}}=2\left(1+\frac{\pi}{2}\right)
$$

and not simply $\Delta \kappa_{\mathrm{sdb}} / \Delta \kappa_{\mathrm{eg}}=2$. The physical origin of this effect is well understood [25,33]: If the two blocks of a symmetric copolymer are decoupled at the membrane, then the end-grafted chains induce a local spontaneous curvature of equal magnitude but opposite sign. Asymmetric membrane inclusions like these redistribute on the membrane and lower the overall bending rigidity of the system. In the limit of small curvatures, the total bending rigidity has been shown to be [30]

$$
\Delta \kappa=\left[a_{\kappa}^{(c)}-4\left(a_{\mathrm{sp}}^{(c)}\right)^{2}\right] k_{B} T \sigma R_{e}^{2}
$$

where the dimensionless amplitudes $a_{\kappa}^{(c)}$ and $a_{\mathrm{sp}}^{(c)}$ are those of a homogeneous distribution of polymers on membranes of constant curvature, with $(\Delta \kappa)_{c}=a_{\kappa}^{(c)} k_{B} T \sigma R_{e}^{2}$ and $\kappa\left(\Delta c_{0}\right)_{c}$ $=a_{\mathrm{sp}}^{(c)} k_{B} T \sigma R_{e}$, as calculated in Refs. [22-24]. Thus the stiffening effect of an end-grafted chain is reduced by a factor of $1 /(1+\pi / 2)$ due to polymer redistribution. For asymmetric diblock copolymers, the strength of the redistribution effect varies with the differences in chain lengths. The general expression (25) for the bending rigidity simplifies in the limit $q \rightarrow 0$ to

$$
\frac{\Delta \kappa_{d b}}{k_{B} T}=\sigma\left(R_{e, M}^{2}+R_{e, N}^{2}\right)\left(\frac{1}{6}+\frac{\pi}{12} \frac{R_{e, N} R_{e, M}}{R_{e, N}^{2}+R_{e, M}^{2}}\right) .
$$

Note that the other two curvature elastic constants, the Gaussian saddle-splay modulus and the spontaneous curvature, are not affected by polymer redistribution [30].

\section{SIMULATIONS}

Simulation studies for polymers attached to fluctuating membranes have been performed for the average shape of the membrane with an end-grafted polymer $[15,16]$, as well as for the pressure exerted by the polymer near the anchor point [18]. The length-scale dependence of second-order effects in the curvature, like the elastic constants $\kappa$ and $\bar{\kappa}$, has so far not been studied by computer simulations.

\section{A. Simulation and evaluation technique}

The Metropolis Monte Carlo Method is used to generate the ensembles, where the RANLUX random number generator $[34,35]$ is employed. The polymer conformations are changed by the pivot algorithm as described in Ref. [22]. With the Fourier-transformed Eq. (6) and the equipartition theorem, the (discretized) relation reads

$$
\kappa_{\text {eff }}(\mathbf{q})=\frac{k_{B} T}{4\left\langle h_{\mathbf{q}} h_{-\mathbf{q}}\right\rangle}\left[\cos \left(q_{x}\right)+\cos \left(q_{y}\right)-2\right]^{-2}
$$

where

$$
h_{\mathbf{q}}=\frac{1}{L} \sum_{q_{x}, q_{y}=0}^{L-1} h_{\boldsymbol{\rho}} \exp \left[\frac{2 \pi i}{L} \boldsymbol{\rho} \cdot \mathbf{q}\right] .
$$

The term $\left\langle h_{\mathbf{q}} h_{-\mathbf{q}}\right\rangle$ denotes the ensemble average of the product of Fourier amplitudes with wave vector q. The bending rigidity can then be directly extracted from the values of the amplitudes $h_{\mathbf{q}}$. In order to keep the relaxation times essentially independent of the wave vector, it is most convenient to update the membrane conformation in Fourier space. However, a transformation to real space is still needed to compare membrane and polymer conformations. This transformation has been implemented using the FFTW fast Fourier transformation library [36].

There are different ways of representing the membrane conformation in real space. One possibility, a kind of "solidon-solid" model, is to define height levels $h(x)=h_{i}$ for $x_{i}$ 

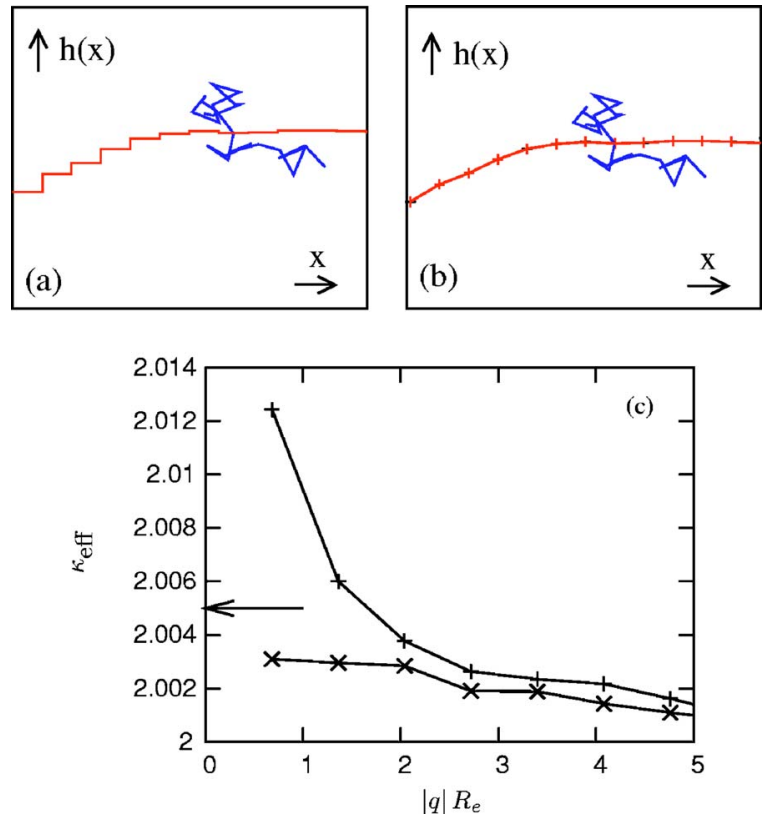

FIG. 5. (Color online) Simulation snapshots for a system of $L$ $=32, N=12$, and $\kappa / k_{B} T=2$, which show the same conformations, but different membrane representations to model the membranepolymer interaction. (a) Solid-on-solid model. (b) Piecewise linear interpolation of height variables. (c) The bending rigidity for the solid-on-solid model strongly increases for $|\mathbf{q}| \rightarrow 0(+)$. The arrow denotes the analytical value for large curvature radii $[23,24]$. For the piecewise interpolation, the bending rigidity converges for $|\mathbf{q}|$ $\rightarrow 0$, but apparently to a too small value $(\times)$.

$-a / 2<x<x_{i}+a / 2$ around each grid point, as in the simple clapper model [see Figs. 2 and 5(a)]. Unfortunately, the bending rigidity $\kappa_{\text {eff }}(\mathbf{q})$, which is extracted from the simulation data, appears to diverge in the limit $|\mathbf{q}| \rightarrow 0$ [compare Fig. 5(c)]. This problem can be traced back to those conformations in which the polymer is located in places where the membrane forms a staircase. The approximation of the membrane shape is obviously very crude in these cases, and membrane fluctuations can be expected to be strongly suppressed by the polymer. This conclusion has been checked with a polymer attached to a membrane of sinusoidal shape $h(x, y) \propto \sin (x)$. If a diblock copolymer is attached at $x=0$, it is located at the mainly planar parts of the membrane and should have a very small influence on the fluctuations. On the other hand, if the polymer is attached at $x=\pi / 2$, it is located in the highly curved parts and its influence on the fluctuations should be most pronounced. However, with the solid-on-solid discretization a strong effect is obtained if the polymer is anchored at $x=0$, and a weak effect in case it is anchored at $x=\pi / 2$. To avoid this "staircase" artifact, a model has been chosen with a linear interpolation between the membrane height variables $h_{i}$ for all subsequent simulations, such that the membrane deformation $h(\boldsymbol{\rho})$ is a continuous function [see Fig. 5(b)]. As shown in Fig. 5(c), in this case the simulation data nicely extrapolate to a finite value in the limit $\mathbf{q} \rightarrow 0$ - to a value that is apparently smaller than the analytic result for Gaussian chains [23]. This deviation is due to discretization effects.

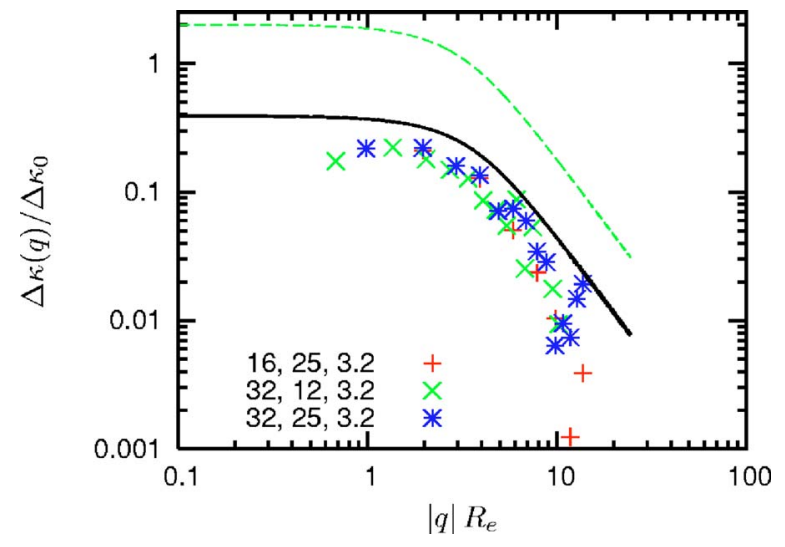

FIG. 6. (Color online) The polymer effect of end-grafted chains attached to one side of the membrane. The simulation data are labeled by the set of $L, N$, and $\kappa / k_{B} T$. The solid line is the analytical result (21), the dashed line the analytical expression (24).

\section{B. Results for ideal end-grafted polymers}

Systems have been investigated with polymer lengths $N$ $=12,25$, and 50 and membrane sizes $L=16,32$, and 64 to extract the length-scale-dependent polymer effect from the simulations (see Fig. 6). The data of this and all following plots are normalized by $\Delta \kappa_{0}=0.21 k_{B} T \sigma R_{e}^{2}$ for ideal and $\Delta \kappa_{0}=0.20 k_{B} T \sigma R_{e}^{2}$ for self-avoiding chains as calculated in Ref. [22] for single chains in the limit $|\mathbf{q}| \rightarrow 0$ and without redistribution effects. In order to extract the universal behavior of Gaussian chains, the simulation data also have to be extrapolated for $N \rightarrow \infty$. It has been shown in Ref. [22] that in the limit $|\mathbf{q}| \rightarrow 0$, the excess bending rigidity $\Delta \kappa(\mathbf{q})$ including the corrections to scaling due to finite chain length $N$ is given by Eq. (27),

$$
\Delta \kappa / k_{B} T=0.213\left(1-0.87 N^{-1 / 2}\right) \sigma R_{e}^{2}
$$

and

$$
\kappa \Delta c_{0} / k_{B} T=0.180\left(1-0.37 N^{-1 / 2}\right) \sigma R_{e} .
$$

The statistical error of the universal amplitude is about 0.005 and of the correction-to-scaling prefactor about 0.01 . We assume that the same corrections occur for finite wave vectors. Therefore, we extrapolate to $N \rightarrow \infty$ by normalizing the simulation results according to Eqs. (27), (31), and (32). Two different scenarios will be discussed in the following: the case of a single chain which is free to move on the membrane and the case of several chains, regularly attached at fixed distances from each other.

For one end-grafted chain, polymer redistribution takes place and the stiffening effect on the bending rigidity is lowered accordingly. In the double-logarithmic plot in Fig. 6, also the two regimes of $\kappa_{\text {eff }}(\mathbf{q})$ can be identified [25]: the "surface-tension regime" with $\Delta \kappa(\mathbf{q}) \propto \mathbf{q}^{-2}$ for large $|\mathbf{q}|$ values, and the bending-rigidity regime with almost constant $\Delta \kappa(\mathbf{q})$ for small $|\mathbf{q}|$. At large $|\mathbf{q}|$ the statistical errors of the simulation data become large since the polymer effect is more than three orders of magnitude smaller than the bending rigidity of the bare membrane. The functional form of the excess bending rigidity obtained from the simulations fol- 


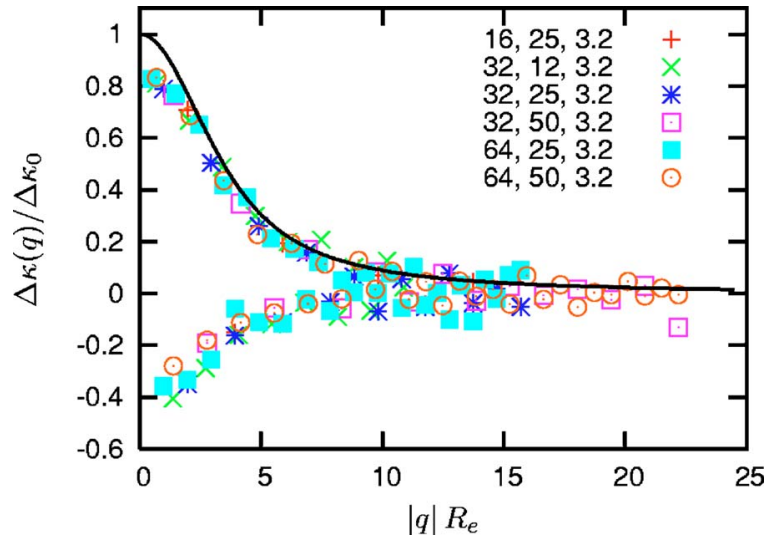

FIG. 7. (Color online) Excess bending rigidity for two endgrafted chains attached at fixed distance $\ell=L a / 2$ to the same side of a fluctuating membrane, as a function of the scaled wave number $|\mathbf{q}| R_{e}$. The data are labeled by $L, N$, and $\kappa / k_{B} T$. The solid line is the analytical result (24) per block of a symmetric diblock copolymer.

lows the analytical prediction (21) very well. However, the polymer effect observed in the simulations is almost a factor of 2 smaller than predicted. We interpret this reduction as an effect due to a local pinch deformation of the membrane near the anchor point $[15,18]$, since it is not observed for symmetric diblocks. Although the analytical calculation contains terms up to second order, the first-order effect of a pinch is not included in the model. The membrane-polymer interaction is replaced by an interaction of the polymer with a planar hard wall and zero anchor distance. A deformation of the membrane-like the formation of a pinch-modifies this interaction. ${ }^{2}$.

In the case of more than one chain attached with regular spacing to the same side of the membrane, several effects are observed. Figure 7 shows the data for two chains on the membrane. First, due to the local spontaneous curvature induced by the polymers, undulations with $q=2 \pi m / \ell$ are favored, where $\ell$ is the distance between the anchor points of the polymers. At the corresponding $q$ values all chains are located on membrane ridges [see Fig. 8(a)]. The undulation amplitudes for these wavelengths are therefore enhanced, and the $\Delta \kappa$ values are negative. Simulation data for four chains per membrane fall on the same curve, but are not shown. Second, for the other wave vectors, the bending rigidity is increased. For an even number of chains, the normalized data for $\Delta \kappa(\mathbf{q})$ in the surface-tension regime is well described by the analytical function for symmetric diblocks. This is no coincidence, since due to the regular spacing, all chains can be grouped into pairs such that the two chains encounter mirror images of their respective local membrane conformations, just like the two blocks of a symmetric copolymer, as illustrated in Fig. 8(b).

\footnotetext{
${ }^{2}$ Contrary to the diblock case, the simulation results for endgrafted chains also depend more strongly on the parameters of the system, e.g., the ratio of polymer length to membrane size, or whether the chain is attached to a membrane grid point or to the middle of a plaquette. Therefore, we do not discuss the relatively small effects of self-avoidance for end-grafted polymers.
}

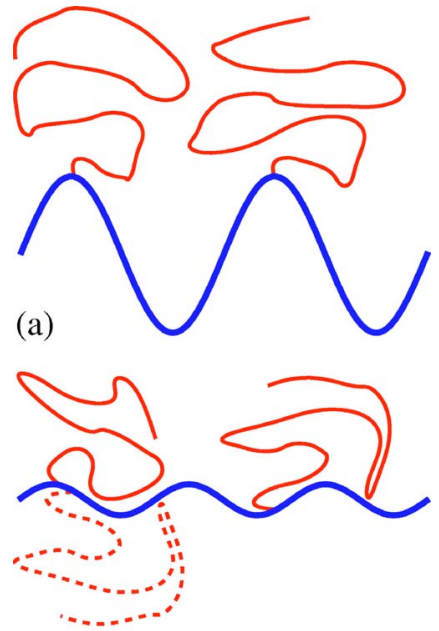

(b)

FIG. 8. (Color online) Two end-grafted chains attached at fixed distance $\ell=L a / 2$ to the same side of a fluctuating membrane. (a) Fluctuations with $q=2 \pi m / \ell$ are enhanced, while (b) for undulation modes with other wave vectors the system behaves just like a membrane with a symmetric diblock copolymer (dashed line).

\section{Results for ideal diblock copolymers}

For the symmetric diblock copolymer chains, redistribution effects and membrane pinches, as discussed for the endgrafted chain, do not occur. Thus a direct comparison of the bending rigidity per chain in the limit $|\mathbf{q}| \rightarrow 0$ with the results in Refs. [22-24] is possible. After normalization of the data according to Eq. (31), the data are very well described by Eq. (24) for the symmetric diblock copolymers (compare Fig. 9). Note that there are no adjustable parameters. This is remarkable in particular since polymers with small bond numbers are used in the simulations, and the correction-to-scaling relation (31), which was obtained in the $|\mathbf{q}| \rightarrow 0$ limit, is applied to all length scales.

The remaining small deviations of the simulation data from the analytical result might be due to discretization or

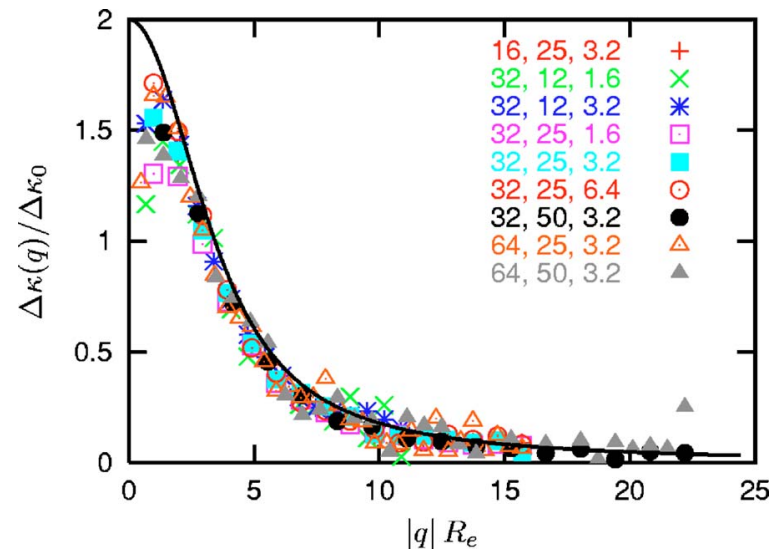

FIG. 9. (Color online) Functional dependence of $\Delta \kappa(\mathbf{q})$ for symmetric ideal diblock copolymers anchored to a fluctuating membrane. The line is the analytical result of Eq. (24); the simulation data are labeled by $L, N$, and $\kappa / k_{B} T$. 


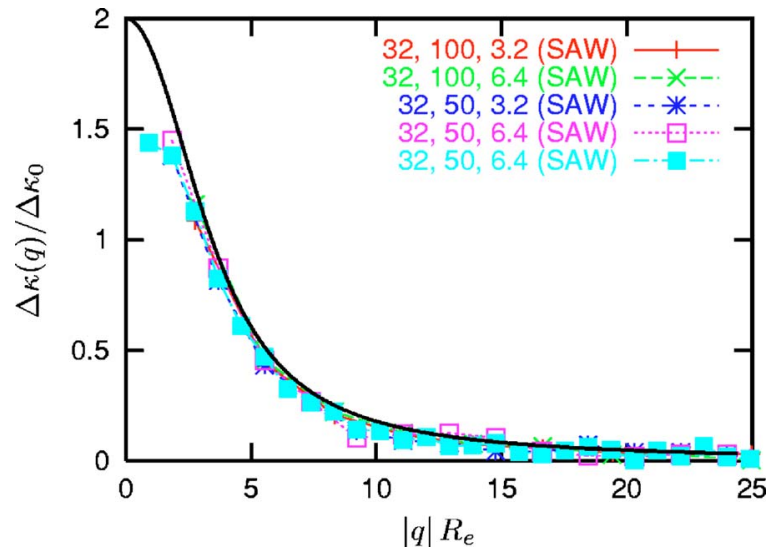

FIG. 10. (Color online) Excess bending rigidity $\Delta \kappa(\mathbf{q})$ for symmetric self-avoiding diblock copolymers anchored to a fluctuating membrane. The simulation data are labeled by $L, N$, and $\kappa / k_{B} T$. The functional form of the data equals the one found for ideal polymer chains, since the line is again the analytical result of Eq. (24) with the self-avoiding $R_{e}$.

supercell effects. Mode-coupling effects seem to be partly responsible for the drop of the polymer effect at low $|\mathbf{q}|$ values, but do not explain the deviation completely [37].

\section{Results for self-avoiding diblock copolymers}

Self-avoidance effects for linear polymer chains have been studied with beads of radius $0.25 a$ attached to the polymer's vertices. In this case, the excess bending rigidity $\Delta \kappa(\mathbf{q})$ due to the polymer effect in the small-curvature limit is given by [22]

$$
\Delta \kappa / k_{B} T=0.20\left(1-0.97 N^{-1 / 2}\right) \sigma R_{e}^{2}
$$

where the statistical errors are of the order 0.01. As for the ideal chains, the simulation data again fall on a single master curve (see Fig. 10). Note that the conformations of selfavoiding chains are much more stretched than those of ideal chains: for a chain of 200 bonds and bead size $0.25 a$, the root-mean-squared end-to-end radius $R_{e}$ as well as the radius of gyration $R_{g}$ are about a factor of 1.5 larger than for the corresponding ideal chain [37]. Surprisingly, with proper normalization according to Eq. (33), self-avoidance seems to affect only the amplitude of the polymer effect. The functional form of the polymer effect on the fluctuation spectrum is again very well described by Eq. (24) with the appropriate end-to-end distance $R_{e}$ for self-avoiding chains. A similar observation for the pressure of an anchored chain on the anchoring wall has been made in Ref. [18]: the scaled pressure distribution is found to remain essentially unchanged when self-avoidance is introduced.

\section{E. Results for star polymers}

The direct effect of polymers on the bending rigidity of a membrane can be enhanced considerably at fixed polymer content by contracting the anchors of several linear chains into one, i.e., by the formation of star polymers. As discussed in Ref. [22] in the limit $|\mathbf{q}| \rightarrow 0$, the membrane stiffening

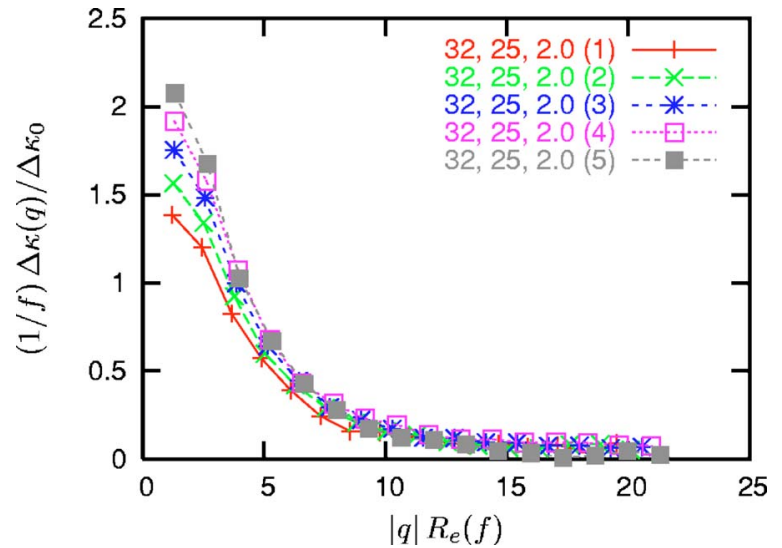

FIG. 11. (Color online) Length-scale-dependent polymerinduced bending rigidity $\Delta \kappa(\mathbf{q})$ for symmetric diblock star polymers, with $f=1, \ldots, 5$ arms on each side of the membrane. One arm consists of $N=25$ bonds and beads of radius $0.25 a$. The simulation data are labeled by $L, N, \kappa_{\mathrm{mem}} / k_{B} T$, and $f$. All amplitudes are normalized by $f \Delta \kappa_{0}$ in order to show the increase of the bending rigidity with star functionality $f$.

increases by a factor of about 1.5 already for a star of functionality $f=5$, compared to five linear chains grafted to different points of the membrane. The observed increase in efficiency is much larger than the corresponding increase of the star radius $R_{e}(f)$ in the bulk. For stars with arms of 25 bonds and beads of radius $0.25 a$, the root-mean-squared extension of the arms increases with increasing functionality as $R_{e}(1)=6.22 a, R_{e}(2)=6.40 a, R_{e}(3)=6.55 a, R_{e}(4)=6.68 a$, and $R_{e}(5)=6.79 a$. The bending rigidities per arm of symmetrically attached stars with $f$ arms on each side of the membrane are plotted in Fig. 11 as a function of $|\mathbf{q}| R_{e}(f)$. We employ here $R_{e}(f)$ as the scale factor, since it is the natural length to characterize the size of star polymers. The polymer effect increases with increasing functionality (compared to an equivalent number if diblock copolymers) not only in the limit $|\mathbf{q}| \rightarrow 0$, but over a wide range of wave vectors (compare Fig. 11).

If the chains are attached only to one side of the membrane, not only the direct effect on the bending rigidity but also the induced spontaneous curvature increases with star functionality. Thus the stiffening is opposed by an increased redistribution effect which softens the membrane. The overall effect on the bending rigidity can be estimated in the limit $|\mathbf{q}| \rightarrow 0$ using Eq. (27). The amplitudes of the polymer effect calculated in Ref. [22], as well as the expected amplitudes for stars grafted only to one side of the membrane, are given in Table II. We expect that in this case star polymers decrease the bending rigidity of the membrane. This behavior is indeed observed in the simulation data plotted in Fig. 12. For sufficiently high functionalities, the polymer effect can easily be large enough to make the total effective bending rigidity, $\kappa_{\text {eff }}(\mathbf{q})=\kappa+\Delta \kappa(\mathbf{q})$, negative for small wave numbers. This implies an instability, at which a permanently corrugated membrane shape becomes favorable.

A reduction of the bending rigidity for two-armed star polymers anchored to a membrane due to the anisotropy of these "membrane inclusions" has been predicted in Ref. [32]. 
TABLE II. Universal amplitudes (per chain) for asymmetrically anchored star polymers of functionality $f$ [22] and overall effective amplitude $a_{\kappa \text {,eff }}$ including polymer redistribution, as estimated from Eq. (27). The effective amplitude is normalized by $a_{\kappa}^{(c)}(1)=0.20$. All amplitudes are given in units of $k_{B} T$. The statistical error of the amplitudes is of the order of $10^{-2}$; thus the error estimate for $a_{\kappa, \text { eff }}(f) / a_{\kappa}^{(c)}(1)$ is given by $0.25 f+1.0\left(\kappa c_{0}\right) f^{2}$. The amplitude $\bar{a}_{\kappa}$ of the saddle-splay modulus $\bar{\kappa}$ is defined in the same way as the amplitude $a_{\kappa}$ of the bending rigidity $\kappa$ [see text just before Eq. (21) or Ref. [22]].

\begin{tabular}{ccccc}
\hline \hline$f$ & $a_{\mathrm{sp}}^{(c)} / f$ & $a_{\kappa}^{(c)} / f$ & $\bar{a}_{\kappa}^{(c)} / f$ & $a_{\kappa, \mathrm{eff}}(f) / a_{\kappa}^{(c)}(1)$ \\
\hline 1 & 0.17 & 0.20 & -0.15 & 0.45 \\
2 & 0.19 & 0.22 & -0.16 & -0.75 \\
3 & 0.22 & 0.26 & -0.17 & -4.5 \\
4 & 0.24 & 0.27 & -0.17 & -12.3 \\
5 & 0.25 & 0.30 & -0.14 & -18.4 \\
\hline \hline
\end{tabular}

This effect is different from the reduction of the bending rigidity due to the polymer asymmetry discussed above. The anisotropy effect should be present for end-grafted as well as symmetrically attached stars with $f=2$ and is expected to become less pronounced for the more isotropic stars with $f$ $>2$. In the data of Fig. 11, a particularly pronounced decrease of the bending rigidity for $f=2$ stars is not observed. The anisotropy effect thus seems to be smaller than estimated in Ref. [32]. This is not unexpected, since the approximation of Ref. [32], to mimic the excluded-volume interaction by a planar, inpenetrable plane between two Gaussian chains, certainly overestimates the mutual repulsion of the chains and therefore induces a too large anisotropy.

\section{SUMMARY AND CONCLUSIONS}

The effects of ideal and self-avoiding linear as well as star polymers on the membrane's fluctuation spectrum have been investigated by means of an analytical Green's function method and by Monte Carlo simulations. We have extended

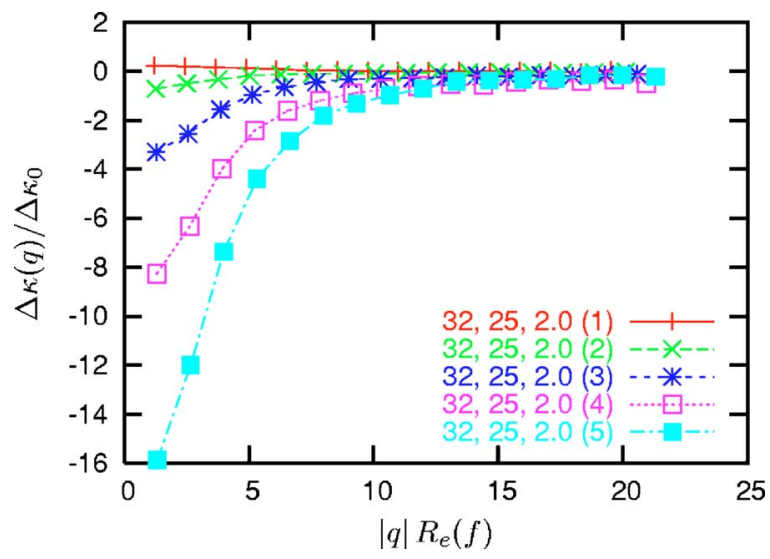

FIG. 12. (Color online) Polymer effect of star polymers grafted at their center to a membrane and having all arms only at one side of the membrane. The simulation data are labeled by $L, N$, $\kappa_{\text {mem }} / k_{B} T$, and $f$. the analytical calculations for end-grafted linear polymers [25] to linear diblock copolymers. The latter system applies to all experimental studies of the polymer-boosting effect in microemulsions. Not only the amplitudes in the limit of large fluctuations but also the functional forms of $\kappa_{\text {eff }}(\mathbf{q})$ differ in both cases.

The effects of self-avoidance have been studied for diblock copolymers and have been shown not to affect the functional dependence of the bending rigidity on the fluctuation length scale, but only the overall amplitude.

Star polymers anchored symmetrically on both sides of the membrane have been found to stiffen the composite membrane. The bending rigidity per arm increases with the functionality over a wide range of wave vectors. Star polymers which are attached only to one side of the membrane strongly decrease the bending rigidity for high functionalities. The decrease is due to a large locally induced spontaneous curvature and the resulting polymer redistribution. It can be large enough to make the total effective bending rigidity negative and thereby lead to a permanently corrugated membrane shape.

\section{ACKNOWLEDGMENTS}

Discussions with E. Eisenriegler, D. Richter, and H. Frielinghaus are gratefully acknowledged.

\section{APPENDIX A: DETAILS OF THE ANALYTICAL CALCULATION FOR END-GRAFTED CHAINS}

We provide here details of the calculations of the partition function for end-grafted chains in Sec. III B and Ref. [25]. Specific formulas of Ref. [38] are quoted for some steps where we consider them helpful. The positions on the membrane are characterized by $\boldsymbol{\rho}=(x, y)$ and a full set of coordinates will be abbreviated by $\mathbf{r}=(x, y, z)$. Following the method of Refs. [25,30,32], the "magic rule" [39]

$$
Z_{N}^{(i)}(\mathbf{r})=\frac{a^{2}}{6} \int_{0}^{N} d n \int d \boldsymbol{\rho}^{\prime} \frac{\partial G_{N-n}^{(0)}}{\partial z}\left(\mathbf{r} ; \boldsymbol{\rho}^{\prime}, 0\right) Z_{n}^{(i)}\left(\boldsymbol{\rho}^{\prime}, 0\right)
$$

is employed. The boundary conditions of Eq. (17), which have to be satisfied for each power in $h$ independently,

$$
\begin{gathered}
Z_{N}^{(0)}(\boldsymbol{\rho}, 0)=0 \\
Z_{N}^{(1)}(\boldsymbol{\rho}, 0)=-h(\boldsymbol{\rho}) \frac{\partial Z_{N}^{(0)}}{\partial z}(\boldsymbol{\rho}, 0), \\
Z_{N}^{(2)}(\boldsymbol{\rho}, 0)=-h(\boldsymbol{\rho}) \frac{\partial Z_{N}^{(1)}}{\partial z}(\boldsymbol{\rho}, 0)-\frac{1}{2} h(\boldsymbol{\rho})^{2} \frac{\partial^{2} Z_{N}^{(0)}}{\partial z^{2}}(\boldsymbol{\rho}, 0)
\end{gathered}
$$

connect the different orders of the perturbation expansion. Note that technically speaking, the boundary conditions are not evaluated for $z=0$ but for $z=\delta$, and the limit $\delta \rightarrow 0$ be taken at a later stage. Thus, starting from the ground state 
$(i=0)$, all orders in the perturbation $(i>1)$ can be calculated.

With the membrane height measured relatively to the height of the anchor point, $h(\boldsymbol{\rho})-h\left(\boldsymbol{\rho}^{\prime}\right)$, the first-order term for a polymer anchored at position $\boldsymbol{\rho}$ reads

$$
\begin{aligned}
& Z_{N}^{(1)}(\mathbf{r})=\lim _{\delta \rightarrow 0}\left(-\frac{a^{2}}{6}\right) \int_{0}^{N} d n \int d \boldsymbol{\rho}^{\prime}\left[h\left(\boldsymbol{\rho}^{\prime}\right)-h(\boldsymbol{\rho})\right] \\
& \times\left.\frac{\partial G_{N-n}^{(0)}}{\partial z}\left(\mathbf{r} ; \boldsymbol{\rho}^{\prime}, \delta\right) \frac{\partial Z_{N}^{(0)}\left(\mathbf{r}^{\prime}\right)}{\partial z^{\prime}}\right|_{z^{\prime}=\delta}
\end{aligned}
$$

and evaluates to

$$
\begin{aligned}
Z_{N}^{(1)}(\mathbf{r})= & -\frac{23^{2}}{\pi a^{4}} z \int d \boldsymbol{\rho}^{\prime}\left[h\left(\boldsymbol{\rho}^{\prime}\right)-h(\boldsymbol{\rho})\right] \\
& \times \mathcal{L}_{N}^{-1}\left[\left(\frac{1}{\sqrt{s k^{3}}}+\frac{1}{k^{2}}\right) e^{-\sqrt{s k}}\right],
\end{aligned}
$$

where $k=(\sqrt{6} / a) \sqrt{\left(x-x^{\prime}\right)^{2}+\left(y-y^{\prime}\right)^{2}+z^{2}}$, and the $n$-integral has been expressed as an inverse Laplace transform $\mathcal{L}^{-1}$ with respect to the variable $s$. After evaluating the transformation, Eq. (A4) reads

$$
\begin{aligned}
Z_{N}^{(1)}(\mathbf{r})= & -\frac{23^{2}}{\pi^{3 / 2} a^{4}} z \int d \boldsymbol{\rho}^{\prime}\left[h\left(\boldsymbol{\rho}^{\prime}\right)-h(\boldsymbol{\rho})\right] \\
& \times\left(\frac{1}{k^{3} N^{1 / 2}}+\frac{1}{2 k N^{3 / 2}}\right) e^{-k^{2} /(4 N)} .
\end{aligned}
$$

The average of $Z_{N}^{(1)}(\mathbf{r})$ over the position $\boldsymbol{\rho}$ of the anchor point vanishes, which can easily be seen since the integrals over the two terms containing $h(\boldsymbol{\rho})$ and $h\left(\boldsymbol{\rho}^{\prime}\right)$, respectively, become identical after an interchange of the integration variables $\boldsymbol{\rho}$ and $\boldsymbol{\rho}^{\prime}$ in the second term.

The derivation of the second-order term is more difficult. Therefore more steps of the calculation are presented, also to serve as an example for the type of calculations required for the diblock copolymers in Sec. III C and Appendix B. The initial expression given by the magic rule and the boundary conditions reads

$$
\begin{aligned}
Z_{N}^{(2)}(\mathbf{r})= & -\frac{a^{2}}{6} \lim _{\delta \rightarrow 0} \int_{0}^{N} d n \int d \boldsymbol{\rho}^{\prime} \frac{\partial G_{N-n}^{(0)}}{\partial z}\left(\mathbf{r} ; \boldsymbol{\rho}^{\prime}, \delta\right)\left(\left.\left[h\left(\boldsymbol{\rho}^{\prime}\right)-h(\boldsymbol{\rho})\right] \frac{\partial Z_{n}^{(1)}\left(\boldsymbol{\rho}^{\prime}, z^{\prime}\right)}{\partial z^{\prime}}\right|_{z^{\prime}=\delta}+\left.\frac{\left[h\left(\boldsymbol{\rho}^{\prime}\right)-h(\boldsymbol{\rho})\right]^{2}}{2} \frac{\partial^{2} Z_{n}^{(0)}\left(\boldsymbol{\rho}^{\prime}, z^{\prime}\right)}{\partial z^{\prime 2}}\right|_{z^{\prime}=\delta}\right) \\
= & \frac{3^{7 / 2}}{2^{3 / 2} \pi^{5 / 2} a^{7}} \lim _{\delta \rightarrow 0} \int d \boldsymbol{\rho}^{\prime} \int_{0}^{N} d n\left[h\left(\boldsymbol{\rho}^{\prime}\right)-h(\boldsymbol{\rho})\right] \frac{1}{(N-n)^{5 / 2}}\left[(z-\delta) e^{-k_{-}^{2} / 4 /(N-n)}+(z+\delta) e^{-k_{+}^{2} / 4 /(N-n)}\right]\left(\int d \boldsymbol{\rho}^{\prime \prime}\left[h\left(\boldsymbol{\rho}^{\prime \prime}\right)-h(\boldsymbol{\rho})\right]\right. \\
& \left.\times\left\{\mathcal{L}_{n}^{-1}\left[\left(\frac{1}{\sqrt{s k^{\prime 3}}}+\frac{1}{k^{\prime 2}}\right) e^{-\sqrt{s k^{\prime}}}\right]-\frac{6}{a^{2}} \delta^{2} \mathcal{L}_{n}^{-1}\left[\left(\frac{3}{\sqrt{s k^{\prime 5}}}+\frac{3}{k^{\prime 4}}+\frac{\sqrt{s}}{k^{\prime 3}}\right) e^{-\sqrt{s k^{\prime}}}\right]\right\}+\frac{a}{3^{1 / 2} 2^{3 / 2} \pi^{1 / 2}}\left[h\left(\boldsymbol{\rho}^{\prime}\right)-h(\boldsymbol{\rho})\right] \frac{\delta}{n^{3 / 2}} e^{-k_{\delta}^{2} / 4 / n}\right)
\end{aligned}
$$

where $\quad k^{\prime}=(\sqrt{6} / a) \sqrt{\left(x^{\prime}-x^{\prime \prime}\right)^{2}+\left(y^{\prime}-y^{\prime \prime}\right)^{2}+\delta^{2}}, \quad k_{ \pm}$ $=(\sqrt{6} / a) \sqrt{\left(x-x^{\prime}\right)^{2}+\left(y-y^{\prime}\right)^{2}+(z \pm \delta)^{2}}$, and $k_{\delta}=(\sqrt{6} / a) \delta$. The inverse Laplace transforms enter via $\partial Z_{n}^{(1)}\left(\boldsymbol{\rho}^{\prime}, z^{\prime}\right) / \partial z^{\prime}$ by use of Eq. (A4). With the help of the convolution Theorem 29.2.8 [38], as well as Eqs. 29.3.84 and 29.2.11, the integral over $n$ can again be evaluated by Laplace transforms. The second-order term then reads

$$
\begin{aligned}
Z_{N}^{(2)}(\mathbf{r})= & \frac{3^{7 / 2} 2^{1 / 2}}{\pi^{2} a^{7}} \lim _{\delta \rightarrow 0} \int d \rho^{\prime}\left[h\left(\boldsymbol{\rho}^{\prime}\right)-h(\boldsymbol{\rho})\right] \iint d \boldsymbol{\rho}^{\prime \prime}\left[h\left(\boldsymbol{\rho}^{\prime \prime}\right)\right. \\
& -h(\boldsymbol{\rho})] \mathcal{L}_{N}^{-1}\left[f_{k}\left(\frac{1}{\sqrt{s k^{\prime 3}}}+\frac{1}{k^{\prime 2}}\right) e^{-\sqrt{s k^{\prime}}}\right] \\
& -\frac{6}{a^{2}} \delta^{2} \int d \boldsymbol{\rho}^{\prime \prime}\left[h\left(\boldsymbol{\rho}^{\prime \prime}\right)-h(\boldsymbol{\rho})\right] \mathcal{L}_{N}^{-1}\left[f _ { k } \left(\frac{3}{\sqrt{s k^{\prime 5}}}+\frac{3}{k^{\prime 4}}\right.\right. \\
& \left.\left.\left.+\frac{\sqrt{s}}{k^{\prime 3}}\right) e^{-\sqrt{s} k^{\prime}}\right]+\frac{\pi a^{2}}{6}\left[h\left(\boldsymbol{\rho}^{\prime}\right)-h(\boldsymbol{\rho})\right] \mathcal{L}_{N}^{-1}\left[\left(f_{k} e^{-\sqrt{s} k} \delta\right)\right]\right\}
\end{aligned}
$$

where

$$
f_{k}=\left[(z-\delta)\left(\frac{\sqrt{s}}{k_{-}^{2}}+\frac{1}{k_{-}^{3}}\right) e^{-\sqrt{s} k_{-}}+(z+\delta)\left(\frac{\sqrt{s}}{k_{+}^{2}}+\frac{1}{k_{+}^{3}}\right) e^{-\sqrt{s} k_{+}}\right] .
$$

The Fourier transformation of Eq. (A7) with respect to $\boldsymbol{\rho}$ $-\boldsymbol{\rho}^{\prime}$ and $\boldsymbol{\rho}^{\prime}-\boldsymbol{\rho}^{\prime \prime}$ can be carried out in polar coordinates using

$$
\int_{0}^{2 \pi} d \phi e^{i q r \cos \phi}=2 \pi J_{0}(q r)
$$

a special case of Eq. 6.637 [40],

$$
\begin{aligned}
& \int_{0}^{\infty} d x x\left(\frac{1}{\beta^{2}+x^{2}}+\frac{1}{\alpha\left(\beta^{2}+x^{2}\right)^{3 / 2}}\right) e^{-\alpha \sqrt{\beta^{2}+x^{2}}} J_{0}(\gamma x) \\
& =\frac{1}{\alpha \beta} e^{-\beta \sqrt{\alpha^{2}+\gamma^{2}}}
\end{aligned}
$$




$$
\begin{aligned}
\int_{0}^{\infty} & d x x\left(\frac{1}{\alpha\left(\beta^{2}+x^{2}\right)^{5 / 2}}+\frac{1}{\left(\beta^{2}+x^{2}\right)^{2}}\right. \\
+ & \left.\frac{\alpha}{3} \frac{1}{\left(\beta^{2}+x^{2}\right)^{3 / 2}}\right) e^{-\alpha \sqrt{\beta^{2}+x^{2}}} J_{0}(\gamma x) \\
= & \frac{1}{3 \alpha \beta^{3}}\left(1+\beta \sqrt{\alpha^{2}+\gamma^{2}}\right) e^{-\beta \sqrt{\alpha^{2}+\gamma^{2}}} .
\end{aligned}
$$

The transformed expression reads

$$
\begin{aligned}
Z_{N}^{(2)}(\mathbf{r})= & -\frac{6}{a^{2}} \int d \boldsymbol{\rho}^{\prime} \int d \boldsymbol{\rho}^{\prime \prime}\left\{\frac { a } { 6 ^ { 1 / 2 } } [ h ( \boldsymbol { \rho } ^ { \prime } ) - h ( \boldsymbol { \rho } ) ] \left[h\left(\boldsymbol{\rho}^{\prime \prime}\right)\right.\right. \\
& -h(\boldsymbol{\rho})] \int \frac{d \mathbf{q}_{1}}{(2 \pi)^{2}} \int \frac{d \mathbf{q}_{2}}{(2 \pi)^{2}} e^{-i \mathbf{q}_{1} \cdot\left(\boldsymbol{\rho}-\boldsymbol{\rho}^{\prime}\right)} e^{-i \mathbf{q}_{2} \cdot\left(\boldsymbol{\rho}^{\prime}-\boldsymbol{\rho}^{\prime \prime}\right)} \\
& \times \mathcal{L}_{N}^{-1}\left[\left(\frac{6}{a^{2}}+\frac{q^{2}}{s}\right)^{1 / 2} e^{-z\left(6 s / a^{2}+q_{1}^{2}\right)^{1 / 2}}\right]-\left[h\left(\boldsymbol{\rho}^{\prime}\right)\right. \\
& -h(\boldsymbol{\rho})]^{2} \int \frac{d \mathbf{q}_{1}}{(2 \pi)^{2}} e^{-i \mathbf{q}_{1} \cdot\left(\boldsymbol{\rho}-\boldsymbol{\rho}^{\prime}\right)} \mathcal{L}_{N}^{-1}\left[\left(e^{\left.\left.\left.-z\left(6 s / a^{2}+q_{1}^{2}\right)^{1 / 2}\right)\right]\right\} .}\right.\right.
\end{aligned}
$$

After replacing $h(\boldsymbol{\rho}), h\left(\boldsymbol{\rho}^{\prime}\right)$, and $h\left(\boldsymbol{\rho}^{\prime \prime}\right)$ by their corresponding Fourier transforms and averaging $Z_{N}^{(2)}$ over all anchor positions $\boldsymbol{\rho},\left\langle Z_{N}^{(2)}\right\rangle$ can be written in the compact form

$$
\begin{aligned}
\left\langle Z_{N}^{(2)}(\boldsymbol{\rho}, d)\right\rangle= & \frac{6^{1 / 2}}{a \mathcal{S}} \int d \mathbf{q} h(\mathbf{q}) h(-\mathbf{q}) \mathcal{L}_{N}^{-1}\left[\left(\frac{6}{a^{2}}+\frac{q^{2}}{s}\right)^{1 / 2}\right. \\
& \left.\left(e^{-d\left(6 s / a^{2}+q^{2}\right)^{1 / 2}}-e^{-d\left(6 s / a^{2}\right)^{1 / 2}}\right)\right]
\end{aligned}
$$

where $\mathcal{S}$ is the total membrane area and $d$ is the anchor length. The inverse Laplace transformation can be evaluated with the help of Eqs. 29.3.36 and 29.3.4 [38] and by exploiting the fact that $d$ is small,

$$
\begin{aligned}
\mathcal{L}_{N}^{-1} & {\left[\sqrt{1+\frac{q^{2} a^{2}}{6 s}}\left(e^{-d \sqrt{6 s / a^{2}+q^{2}}}-e^{-d \sqrt{6 s / a}}\right)\right] } \\
= & \mathcal{L}_{N}^{-1}\left[\sqrt{1+\frac{q^{2} a^{2}}{6 s}}\left(d \sqrt{\frac{6 s}{a^{2}}}-d \sqrt{\frac{6 s}{a^{2}}+q^{2}}\right)\right] \\
= & \mathcal{L}_{N}^{-1}\left[d \sqrt{\frac{6}{a^{2}}}\left(\sqrt{s+\frac{q^{2} a^{2}}{6}}-\sqrt{6}\right)+d \sqrt{\frac{a^{2}}{6}} \frac{q^{2}}{\sqrt{s}}\right]
\end{aligned}
$$

$$
=\frac{d a^{2}}{6^{1 / 2} \pi^{1 / 2}}\left(\frac{3}{R_{e}^{3}}\left(1-e^{-q^{2} R_{e}^{2} / 6}\right)+\frac{q^{2}}{R_{e}}\right) .
$$

The final results for the averaged different orders of the partition function read

$$
\begin{aligned}
&\left\langle Z_{N}^{(0)}(\boldsymbol{\rho}, d)\right\rangle=\frac{6^{1 / 2} d}{\pi^{1 / 2} R_{e}}+\frac{1}{\mathcal{S}} \int \frac{d \mathbf{q}}{(2 \pi)^{2}} h(\mathbf{q}) h(-\mathbf{q}) \frac{3^{1 / 2} q^{2} d}{(2 \pi)^{1 / 2} R_{e}}, \\
&\left\langle Z_{N}^{(1)}(\boldsymbol{\rho}, d)\right\rangle=0, \\
&\left\langle Z_{N}^{(2)}(\boldsymbol{\rho}, d)\right\rangle= \frac{1}{\mathcal{S}} \int \frac{d \mathbf{q}}{(2 \pi)^{2}} h(\mathbf{q}) h(-\mathbf{q}) \\
& \times\left(\frac{2^{1 / 2} 3^{3 / 2} d}{\pi^{1 / 2} R_{e}^{3}}\left(1-e^{-q^{2} R_{e}^{2} / 6}\right)-\frac{6^{1 / 2} q^{2} d}{\pi^{1 / 2} R_{e}}\right) .
\end{aligned}
$$

Note that the term of zeroth order in the height fluctuations contributes to the effective bending rigidity due to the excess area by the membrane fluctuations compared to the reference plane,

$$
\langle\cdots\rangle=\frac{1}{\mathcal{S}} \int d S \cdots=\frac{1}{\mathcal{S}} \int d \boldsymbol{\rho} \sqrt{1+(\nabla h)^{2}} \cdots .
$$

\section{APPENDIX B: DETAILS OF THE ANALYTICAL CALCULATION FOR SYMMETRIC DIBLOCK COPOLYMERS}

For diblock copolymers, the expressions for the partition functions of the polymers above and below the membrane are coupled,

$$
\begin{aligned}
\left\langle Z_{N,+} Z_{N,-}\right\rangle= & \left\langle Z_{N,+}^{(0)} Z_{N,-}^{(0)}+Z_{N,+}^{(0)} Z_{N,-}^{(1)}+Z_{N,+}^{(0)} Z_{N,-}^{(2)}+Z_{N,+}^{(1)} Z_{N,-}^{(0)}\right. \\
& \left.+Z_{N,+}^{(1)} Z_{N,-}^{(1)}+Z_{N,+}^{(2)} Z_{N,-}^{(0)}\right\rangle .
\end{aligned}
$$

Compared to end-grafted polymers the anchor distance $d$ appears on both sides of the membrane and thus terms up to order $d^{2}$ have to be taken into account. Apart from that, the calculation is rather straightforward and can be done analogously to the calculation in Appendix A. The additional inverse Laplace transformation due to the $Z_{N}^{(1)} Z_{N}^{(1)}$ term can again be evaluated for small $d$. Using Eqs. 29.2.48 and 29.3.84 [38] we find

$$
\begin{aligned}
\mathcal{L}_{N}^{-1}\left[\frac{1}{s^{1 / 2}} e^{-d \sqrt{6 s / a^{2}+q^{2}}}\right]= & \mathcal{L}_{N}^{-1}\left[\frac{1}{s^{1 / 2}}\left(e^{-d \sqrt{6 s / a^{2}+q^{2}}}-e^{-d \sqrt{6 s / a^{2}}}\right)+\frac{1}{s^{1 / 2}} e^{-d \sqrt{6 s / a^{2}}}\right]=\mathcal{L}_{N}^{-1}\left[-d \sqrt{\frac{6}{a^{2}}}\left(\frac{\sqrt{s+q^{2} a^{2} / 6}}{\sqrt{s}}-1\right)+\frac{d^{2} q^{2}}{2 \sqrt{s}}\right. \\
& \left.+\frac{1}{\sqrt{s}} e^{-d \sqrt{6 s / a^{2}}}\right]=-d \sqrt{\frac{6}{a^{2}}} \frac{q^{2} a^{2}}{12} e^{-q^{2} a^{2} N / 12}\left[I_{1}\left(\frac{q^{2} a^{2}}{12} N\right)+I_{0}\left(\frac{q^{2} a^{2}}{12} N\right)\right]+\frac{d^{2} q^{2}}{2 \sqrt{\pi}} \sqrt{N}+\frac{1}{\sqrt{\pi}} \sqrt{N} e^{-3 d^{2} /\left(2 a^{2} N\right)} .
\end{aligned}
$$


The terms containing Bessel functions are responsible for the different functional behavior compared to end-grafted chains. The final results of the single terms in Eq. (B1) read

$$
\left\langle Z_{N,+}^{(0)} Z_{N,-}^{(0)}\right\rangle=\frac{6 d^{2}}{\pi R_{e}^{2}}+\frac{6}{\mathcal{S}} \int \frac{d \mathbf{q}}{(2 \pi)^{2}} h(\mathbf{q}) h(-\mathbf{q}) \frac{q^{2} d^{2}}{\pi R_{e}^{2}},
$$

$$
\left\langle Z_{N,+}^{(0)} Z_{N,-}^{(1)}\right\rangle=\left\langle Z_{N,+}^{(1)} Z_{N,-}^{(0)}\right\rangle=0
$$

$$
\begin{aligned}
& \left\langle Z_{N,+}^{(0)} Z_{N,-}^{(2)}\right\rangle=\left\langle Z_{N,+}^{(2)} Z_{N,-}^{(0)}\right\rangle=\frac{6}{\mathcal{S}} \int \frac{d \mathbf{q}}{(2 \pi)^{2}} h(\mathbf{q}) h(-\mathbf{q}) \\
& \times\left(\frac{6 d^{2}}{2 \pi R_{e}^{4}}\left(1-e^{-q^{2} R_{e}^{2} / 6}\right)-\frac{d^{2} q^{2}}{\pi R_{e}^{2}}\right), \\
& \left\langle Z_{N,+}^{(1)} Z_{N,-}^{(1)}\right\rangle=-\frac{1}{\mathcal{S}} \int \frac{d \mathbf{q}}{(2 \pi)^{2}} h(\mathbf{q}) h(-\mathbf{q}) \frac{q^{4} d^{2}}{4} \\
& \times e^{-q^{2} R_{e}^{2} / 6}\left[I_{1}\left(q^{2} R_{e}^{2} / 12\right)+I_{0}\left(q^{2} R_{e}^{2} / 12\right)\right]^{2}
\end{aligned}
$$

up to order $\mathcal{O}\left(h^{2}\right)$.
[1] B. Jakobs et al., Langmuir 15, 6707 (1999).

[2] H. E. Warriner et al., Science 271, 969 (1996).

[3] D. D. Lasic, Angew. Chem., Int. Ed. Engl. 33, 1685 (1994).

[4] A. Zilker, H. Engelhardt, and E. Sackmann, J. Phys. (France) 48, 2139 (1987).

[5] D. E. Discher, N. Mohantes, and E. A. Evans, Science 266, 1032 (1994).

[6] N. Gov, A. G. Zilman, and S. Safran, Phys. Rev. Lett. 90, 228101 (2003).

[7] E. Evans and W. Rawicz, Phys. Rev. Lett. 79, 2379 (1997).

[8] H. Endo, J. Allgaier, G. Gompper, B. Jakobs, M. Monkenbusch, D. Richter, T. Sottmann, and R. Strey, Phys. Rev. Lett. 85, 102 (2000).

[9] H. Endo et al., J. Chem. Phys. 115, 580 (2001).

[10] F. Castro-Roman, G. Porte, and C. Ligoure, Phys. Rev. Lett. 82, 109 (1999).

[11] C. Hiergeist, V. A. Indrani, and R. Lipowsky, Europhys. Lett. 36, 491 (1996).

[12] S. T. Milner and T. A. Witten, J. Phys. (France) 49, 1951 (1988).

[13] S. Alexander, J. Phys. (France) 38, 983 (1977).

[14] R. Lipowsky, Europhys. Lett. 30, 197 (1995).

[15] M. Breidenich, R. R. Netz, and R. Lipowsky, Europhys. Lett. 49, 431 (2000).

[16] M. Breidenich, R. R. Netz, and R. Lipowsky, Eur. Phys. J. E 5, 403 (2001).

[17] T. Bickel, C. Marques, and C. Jeppesen, Phys. Rev. E 62, 1124 (2000).

[18] T. Bickel, C. Jeppesen, and C. M. Marques, Eur. Phys. J. E 4, 33 (2001).

[19] P. B. Canham, J. Theor. Biol. 26, 61 (1970).

[20] W. Helfrich, Z. Naturforsch. C 28, 693 (1973).

[21] E. A. Evans, Biophys. J. 14, 923 (1974).
[22] T. Auth and G. Gompper, Phys. Rev. E 68, 051801 (2003).

[23] E. Eisenriegler, A. Hanke, and S. Dietrich, Phys. Rev. E 54, 1134 (1996).

[24] C. Hiergeist and R. Lipowsky, J. Phys. II 6, 1465 (1996).

[25] T. Bickel and C. M. Marques, Eur. Phys. J. E 9, 349 (2002).

[26] G. Gompper et al., Europhys. Lett. 56, 683 (2001).

[27] F. David, in Statistical Mechanics of Membranes and Surfaces, edited by S. W. D. Nelson, T. Piran (World Scientific, Singapore, 1989), pp. 157-223.

[28] P. Grassberger, P. Sutter, and L. Schäfer, J. Phys. A 30, 7039 (1997).

[29] G. Gompper and D. M. Kroll, Europhys. Lett. 9, 59 (1989).

[30] T. Bickel, Ph.D. thesis, Université Louis Pasteur Strasbourg I, 2001 (unpublished).

[31] E. Eisenriegler, Polymers Near Surfaces: Conformation Properties and Relation to Critical Phenomena (World Scientific, Singapore, 1993).

[32] C. M. Marques and J. B. Fournier, Europhys. Lett. 35, 361 (1996).

[33] S. Leibler, J. Phys. (France) 47, 507 (1986).

[34] F. James, Comput. Phys. Commun. 79, 111 (1994).

[35] F. James, Comput. Phys. Commun. 97, 357 (1996).

[36] M. Frigo and S. G. Johnson, in Proceedings of the International Conference on 1998 IEEE Acoustics Speech and Signal Processing (IEEE, Los Alamitos, CA, 1998), Vol. 3, pp. 13811384.

[37] T. Auth, Ph.D. thesis, Universität zu Köln, 2003 (unpublished).

[38] Handbook of Mathematical Functions, 9th ed. edited by M. Abramowitz and I. A. Stegun, (Dover, New York, 1972).

[39] G. Barton, Elements of Green's Functions and Propagation (Clarendon Press, Oxford, 1989).

[40] I. S. Gradstein and I. M. Ryshik, Tables of Series, Products and Integrals (Harri Deutsch, Frankfurt/Main, 1981). 